\title{
Studies of a general flat space/boson star transition model in a box through a language similar to holographic superconductors
}

\author{
Yan Peng \\ School of Mathematical Sciences, Qufu Normal University, \\ Qufu, Shandong 273165, China \\ E-mail: yanpengphy@163.com
}

ABSTRACT: We study a general flat space/boson star transition model in quasi-local ensemble through approaches familiar from holographic superconductor theories. We manage to find a parameter $\psi_{2}$, which is proved to be useful in disclosing properties of phase transitions. In this work, we explore effects of the scalar mass, scalar charge and Stückelberg mechanism on the critical phase transition points and the order of transitions mainly from behaviors of the parameter $\psi_{2}$. We mention that properties of transitions in quasi-local gravity are strikingly similar to those in holographic superconductor models. We also obtain an analytical relation $\psi_{2} \propto\left(\mu-\mu_{c}\right)^{1 / 2}$, which also holds for the condensed scalar operator in the holographic insulator/superconductor system in accordance with mean field theories.

KEYWORDS: AdS-CFT Correspondence, Holography and condensed matter physics (AdS/CMT)

ArXIV EPRINT: 1705.08694 


\section{Contents}

1 Introduction 1

2 Equations of motion and boundary conditions 2

3 Properties of phase transitions in a box 3

3.1 Scalar condensation with various $q$ and $m^{2} \quad 3$

$\begin{array}{ll}3.2 & \text { Phase transitions with Stückelberg mechanism }\end{array}$

$\begin{array}{lll}4 & \text { Conclusions } & 7\end{array}$

\section{Introduction}

The schwarzschild black holes in flat space with negative specific heat cannot be in equilibrium with the thermal environment since there is inevitable Hawking radiation. For this case, York and other authors provided a way to bypass this problem by putting the black hole in a box to construct a quasi-local thermodynamic ensemble $[1,2]$. In contrast, the AdS black holes are usually thermodynamically stable since in a certain sense the AdS boundary plays a role of the box condition [3]. With the interest of the AdS/CFT correspondence [4-6], the AdS gravity has attracted a lot of attentions, such as there are many literatures about holographic superconductors constructed in the AdS spacetime [7]-[31].

In the Einstein-Maxwell theory, it was shown that phase structures of the system in a box is similar to those of the AdS gravity [32-34]. Then, it is very interesting to include a scalar field to further compare the similarity between transitions in a box and those of the holographic superconductor system. Recently, P. Basu, C. Krishnan and P.N.B. Subramanian initiated a thermodynamic study of such systems constructed by a charged scalar field coupled to electromagnetic field in asymptotic flat space with box boundary conditions [35]. Besides the flat space and normal RN black hole, it also admits hairy solutions of boson star and hairy RN black hole in this model. These two types of hairy solutions are thermodynamically stable for certain region of parameters. Firstly, it provides a way to evade the flat space no-hair theorems. The more interesting result is that the overall phase structure of the system in a box is strikingly similar to that of holographic superconductors in AdS gravity background. For example, there is flat space/black hole transitions corresponding to the classical Hawking-Page transition in AdS gravity [3]. And this model also admits black hole/hairy black hole transitions similar to those in holographic theories. In addition, there are flat space/boson star transitions corresponding to holographic insulator/superconductor and global AdS/boson star systems in AdS gravity [36, 37]. As a further step along this line, it will be very interesting to study phase transitions in a box with condensation diagrams from asymptotic behaviors 
of the scalar field on the boundary, which is in a language more familiar from holographic superconductors in AdS gravity.

On the other side, it is meaningful to generalize the flat space/boson star model in [35] by including a non-zero scalar mass and examine how the scalar mass will affect the critical phase transition points, which has been studied in holographic models [38-40]. Since there is only second order flat space/boson star phase transition in [35] for large charge of the scalar field, it is interesting to extend this discussion to smaller charge as it has been proved that there is first order holographic insulator/superconductor phase transitions for very small charge [37]. As is well known in [41-47], Stückelberg mechanism usually triggers first order holographic phase transitions, so it is meaningful to examine whether the Stückelberg mechanism could trigger first order phase transitions in quasi-local gravity. From another aspect, hairy black holes and boson stars in a box were constructed and dynamical properties of phases have been investigated in [48, 49]. In addition, it was shown in [50] that hairy black holes really represent the end-point of the superradiant instability of RN black holes in a box. So it is also meaningful to study properties of gravity systems in a box from the thermodynamic aspects.

The next sections are planed as follows. In section 2 , we construct the general flat space/boson star phase transition model in a box beyond the probe limit. In section 3, we manage to find a simple way to describe phase transitions and observe various types of transitions by choosing different model parameters of the scalar mass, scalar charge and Stückelberg mechanism. We summarize our main results in the last section.

\section{Equations of motion and boundary conditions}

We begin with the transition model constructed by a charged scalar field coupled to a Maxwell field in the background of four dimensional asymptotic flat spacetime with a fixed radial coordinate $r=r_{b}$ as the time-like box boundary. And the general Lagrange density in Stückelberg form reads [35, 41, 44]:

$$
\mathcal{L}=R-F^{M N} F_{M N}-(\partial \psi)^{2}-F(\psi)\left(\partial \theta-q A_{\mu}\right)^{2}-m^{2} \psi^{2},
$$

where $\mathrm{q}$ and $m$ are the charge and mass of the scalar field respectively. $A_{M}$ stands for the ordinary Maxwell field and $F(\psi)$ is an arbitrary function of $\psi$. In this work, we will adopt the form that $F(\psi)=\psi^{2}+q^{2} C_{4} \psi^{4}$ in the following calculation [44]. $C_{4}=0$ corresponds to the usual transition model in of subsection 3.1 and when $C_{4} \neq 0$, we examine effects of Stückelberg mechanism on transitions in subsection 3.2. Using the gauge symmetry $A_{\mu} \rightarrow A_{\mu}+\partial \alpha, \theta \rightarrow \theta+\alpha$, we can set $\theta=0$ without loss of generality.

For simplicity, we study matter fields with only radial dependence in the form

$$
A=\phi(r) d t, \quad \psi=\psi(r) .
$$

Considering the matter fields' backreaction on the metric, we take the deformed four dimensional boson star solution as [35]

$$
d s^{2}=-g(r) h(r) d t^{2}+\frac{d r^{2}}{g(r)}+r^{2}\left(d \theta^{2}+\sin ^{2} \theta d \varphi^{2}\right) .
$$


From above assumptions, we obtain equations of motion as

$$
\begin{aligned}
\frac{1}{2} \psi^{\prime}(r)^{2}+\frac{g^{\prime}(r)}{r g(r)}+\frac{q^{2} F(\psi) \phi(r)^{2}}{2 g(r)^{2} h(r)}+\frac{\phi^{\prime}(r)^{2}}{g(r) h(r)}-\frac{1}{r^{2} g(r)}+\frac{1}{r^{2}}+\frac{m^{2}}{2 g} \psi^{2} & =0, \\
h^{\prime}(r)-r h(r) \psi^{\prime}(r)^{2}-\frac{q^{2} r F(\psi) \phi(r)^{2}}{g(r)^{2}} & =0, \\
\phi^{\prime \prime}+\frac{2 \phi^{\prime}(r)}{r}-\frac{h^{\prime}(r) \phi^{\prime}(r)}{2 h(r)}-\frac{q^{2} F(\psi) \phi(r)}{2 g(r)} & =0, \\
\psi^{\prime \prime}(r)+\frac{g^{\prime}(r) \psi^{\prime}(r)}{g(r)}+\frac{h^{\prime}(r) \psi^{\prime}(r)}{2 h(r)}+\frac{2 \psi^{\prime}(r)}{r}+\frac{q^{2} F^{\prime}(\psi) \phi(r)^{2}}{2 g(r)^{2} h(r)}-\frac{m^{2}}{g} \psi & =0,
\end{aligned}
$$

Where $F^{\prime}(\psi)=\frac{d F(\psi)}{d \psi}=2 \psi+4 q^{2} C_{4} \psi^{3}$. These equations are nonlinear and coupled, so we have to use the shooting method to integrate the equations from $r=0$ to box boundary $r=r_{b}$ to search for the numerical solutions satisfying boundary conditions. Around $r=0$, the solutions can be expanded as [35]

$$
\begin{aligned}
& \psi(r)=a+b r^{2}+\cdots, \\
& \phi(r)=a a+b b r^{2}+\cdots, \\
& g(r)=1+A r^{2}+\cdots, \\
& h(r)=A A+B B r^{2}+\cdots,
\end{aligned}
$$

where the dots denote higher order terms. Putting these expansions into equations of motion and considering leading terms, we have three independent parameters $a, a a$ and $A A$ left to describe the solutions. With the rescaling $r \rightarrow a r$, we could set $r_{b}=1$. Near the box boundary $(r=1)$, the asymptotic behaviors of the matter fields are

$$
\begin{aligned}
& \psi \rightarrow \psi_{1}+\psi_{2}(1-r)+\cdots, \\
& \phi \rightarrow \phi_{1}+\phi_{2}(1-r)+\cdots,
\end{aligned}
$$

with $\mu=\phi(1)=\phi_{1}$ as the critical chemical potential. We will also make a transformation to take $g_{t t}(1)=1$ with the symmetry $h \rightarrow a^{2} h, \phi \rightarrow \phi, t \rightarrow \frac{t}{a}$ [35]. Since we impose a mirror boundary conditions for the scalar field as $\psi\left(r_{b}\right)=0$, we have to set $\psi_{1}=0$ and try to use $\psi_{2}$ to describe the phase transition, which is similar to approaches in holographic superconductor theories. We will show in the following section that $\psi_{2}$ is a good probe to properties of phase transitions in quasi-local ensemble. We also point out that our box boundary condition is independent of the mass of scalar fields, which is different from that in holographic superconductor theories where asymptotic behaviors of scalar fields at infinity boundary usually depend on the mass.

\section{Properties of phase transitions in a box}

\subsection{Scalar condensation with various $q$ and $m^{2}$}

In this subsection, we firstly show the numerical boson star solutions in figure 1 . In the left panel, the scalar field decreases as approaching the box boundary and at the boundary 

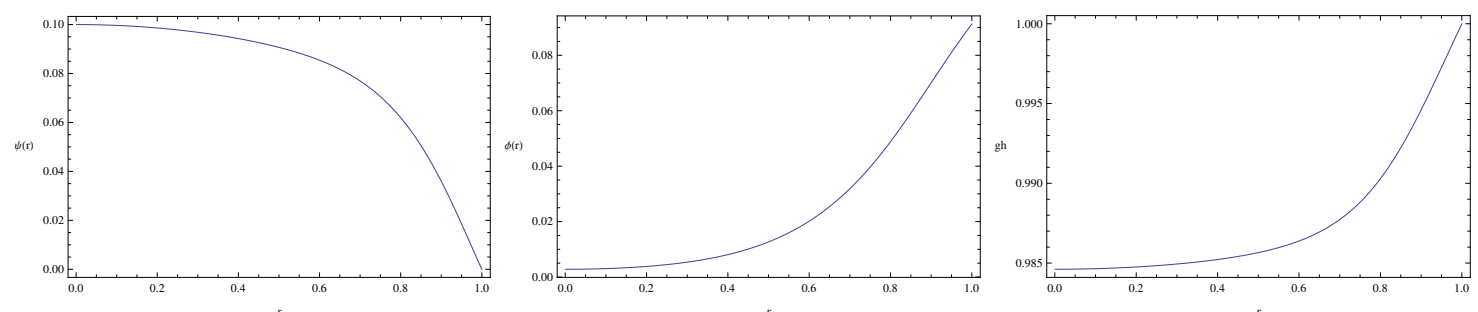

Figure 1. (Color online) We plot solutions as a function of the radial coordinate $r$ with $q=100$, $m^{2}=-2, C_{4}=0$ and $\psi(0)=0.1$. The left panel shows behaviors of $\psi(r)$, the middle panel corresponds to the vector field $\phi(r)$ and the right panel represents the values of $g(r) h(r)=-g_{t t}(r)$.
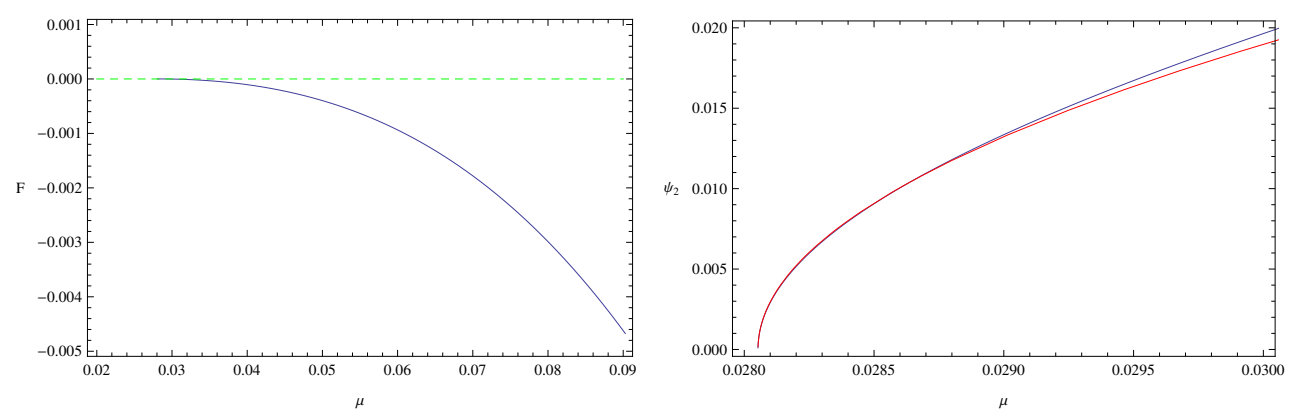

Figure 2. (Color online) We plot the free energy as a function of chemical potential in the left panel with $q=100, m^{2}=-2, C_{4}=0$ and the right panel is with behaviors of $\psi_{2}$. The solid blue line represents boson star phases and the solid red line corresponds to $\psi_{2} \approx 0.430\left(\mu-\mu_{c}\right)^{1 / 2}$ with $\mu_{c}=0.02806$. In addition, the dashed line of $F=0$ in the left panel shows the free energy of the flat space.

there is $\psi\left(r_{b}\right)=0$. In the middle panel, the vector field increases as a function of the radial coordinate. We also show behaviors of the metric solutions $g(r) h(r)=-g_{t t}(r)$ in the right panel. When neglecting the matter fields' backreaction on the metric, we will have $g(r) h(r)=1$ and in contrast, the curves in the right panel show that the metric is deformed by matter fields when including backreaction.

It is known that free energy can be used to determine the critical phase transition points and also the order of transitions. The authors in [35] have proposed a way to calculate the free energy of the system by adding a Gibbons-Hawking-York term on the boundary of the box. After subtracting the free energy of the flat space, we arrive at a formula for the free energy of boson star expressed by the metric solutions and the box boundary radial coordinate as [35]

$$
\begin{aligned}
F= & \frac{1}{\sqrt{g\left(r_{b}\right) h\left(r_{b}\right)}}\left(-\frac{1}{2} \int_{0}^{r_{b}} \sqrt{h(r)} d r-\frac{r_{b}}{2} g\left(r_{b}\right) \sqrt{h\left(r_{b}\right)}-\frac{r_{b}^{2}}{4} g^{\prime}\left(r_{b}\right) \sqrt{h\left(r_{b}\right)}-\frac{r_{b}^{2}}{4} \frac{g\left(r_{b}\right)}{\sqrt{h\left(r_{b}\right)}} h^{\prime}\left(r_{b}\right)\right) \\
& -\left(-r_{b}\right) .
\end{aligned}
$$

We plot the free energy in the case with $q=100, m^{2}=-2$ and $C_{4}=0$ in the left panel of figure 2. Here, we take $q=100$ in order to compare our data with results in [35]. For every fixed value of the chemical potential, we can choose only one phase. Following the 
lowest free energy, it can be seen from the left panel that the solid blue line with a critical chemical potential $\mu_{c}=0.02806$ is physical. Since the free energy is smooth as a function of the chemical potential around $\mu_{c}=0.02806$, the flat space and boson star transition is of the second order. With various charge $q$, we find that there is only second order phase transitions for large charge $q>4.5$, which is qualitatively the same to corresponding properties in holographic insulator/superconductor systems [37].

Inspired by the holographic superconductor theory, we also want to disclose properties of transitions from condensation diagram directly related to the scalar field. We choose to study $\psi_{2}$ as a function of $\mu$ in cases of $q=100, m^{2}=-2$ and $C_{4}=0$ in the right panel of figure 2 with solid blue line. It can be seen from the right panel that $\psi_{2}$ increases as we choose a larger chemical potential, which is qualitatively the same to properties in the holographic insulator/superconductor transition [12,37]. We also find a critical chemical potential $\mu=0.02806$ in the right panel equal to $\mu_{c}=0.02806$ in the left panel, above which the parameter $\psi_{2}$ becomes nonzero. This implies that the parameter $\psi_{2}$ can be used to determine the critical chemical potential of the transition.

By fitting the numerical data, we also obtain an analytical relation $\psi_{2} \propto\left(\mu-\mu_{c}\right)^{1 / 2}$, which also holds in the holographic insulator/superconductor transitions in accordance with mean fields theories [13]. We have plotted the fitting formula $\psi_{2} \approx 0.430\left(\mu-\mu_{c}\right)^{1 / 2}$ with $\mu_{c}=0.02806$ in the right panel of figure 2 with red solid line. It is clearly that the red solid line almost coincides with the $\psi_{2} \sim \mu$ diagram in solid blue line around the phase transition points. It seems that the parameter $\psi_{2}$ plays a role strikingly similar to the scalar operator in holographic insulator/superconductor theories.

For every set of parameters, we obtain a critical chemical potential $\mu_{c}$, above which the flat space gives way to the boson star phases. By choosing $q=100, C_{4}=0$ and various scalar mass $m^{2}$, we disclose effects of the scalar mass on the critical chemical potential $\mu_{c}$ in the left panel of figure 3. It can be easily seen from the left panel that $\mu_{c}$ is larger as we choose a larger $m^{2}$. With more calculations, we go on to plot the critical chemical potential $\mu_{c}$ as a function of $m^{2}$ in the right panel of figure 3. It can be easily seen from the picture that $\mu_{c}$ is almost in linear with respect to the scalar mass $m^{2}$. By fitting the data, we find $\mu_{c} \approx \frac{\pi}{100}+0.001596 * m^{2}$. When $m^{2}=0$, it returns to $\mu_{c}=\frac{\pi}{q}$ in cases of zero scalar mass [35]. That is to say $\mu_{c}$ increases as we choose a less negative scalar mass or larger mass makes the second order phase transitions more difficult to happen, which is in qualitatively accordance with the corresponding properties in holographic insulator/superconductor theories.

Now, we turn to study properties of phase transitions with small charge in our flat space/boson star transition model. In the left panel of figure 4, in cases of $q=4, m^{2}=-2$ and $C_{4}=0$, the free energy has a jump at the critical phase transition points $\mu_{c}=0.6844$ implying that there is a first order phase transitions. We also show $\psi_{2}$ as a function of chemical potential in the right panel with $q=4, m^{2}=-2$ and $C_{4}=0$. It can be seen that $\psi_{2}$ has a jump at the threshold value $\mu_{c}=0.6844$. In other words, $\psi_{2}$ can be used to determine the threshold phase transition points and also the order of transitions. With more calculations, we conclude that small charge $q<4.5$ could trigger first order flat space/boson star transitions similar to cases in holographic insulator/superconductor system [37]. 

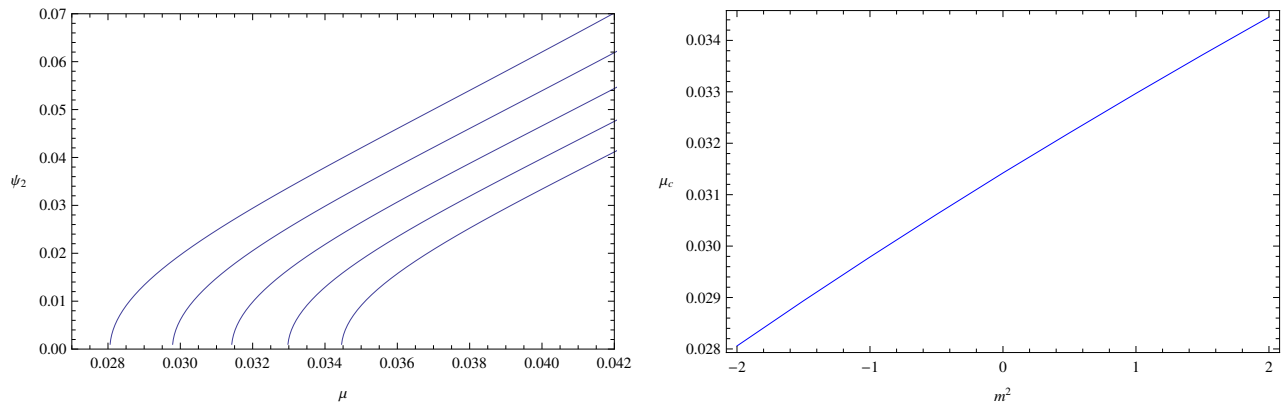

Figure 3. (Color online) In the left panel, we show $\psi_{2}$ as a functions of $\mu$ with $q=100, C_{4}=0$ and various mass $m^{2}$ from left to right as: $m^{2}=-2, m^{2}=-1, m^{2}=0, m^{2}=1$ and $m^{2}=2$. In the right panel, we plot the critical chemical potential $\mu_{c}$ as a function of the scalar mass $m^{2}$ with $q=100$ and $C_{4}=0$.
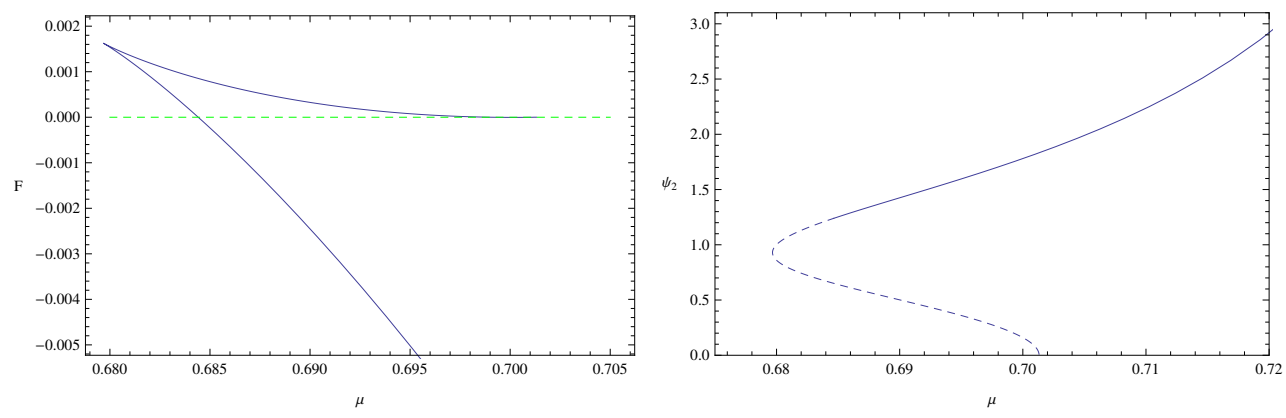

Figure 4. (Color online) We show phases with $q=4, m^{2}=-2$ and $C_{4}=0$. In the left panel, we plot the free energy of boson star as a function of the chemical potential with solid blue curves and the dashed green curves of $F=0$ is with the flat space. The solid blue line in the right panel represents behaviors of parameter $\psi_{2}$ in the thermodynamically stable phases and the dashed blue line in the right panel are with thermodynamically unstable phases.

\subsection{Phase transitions with Stückelberg mechanism}

It is known that holographic superconductor models with Stückelberg mechanism usually allows first order transitions to occur for certain region of parameters in the background of AdS gravity [41-47]. Now we turn to examine whether the Stückelberg mechanism could trigger first order phase transitions for large charge, where there is only second order transitions without Stückelberg mechanism for $q>4.5$ in subsection 3.1. We show the free energy with respect to the chemical potential $\mu$ in the left panel of figure 5 in cases of $q=100, m^{2}=-2$ and $C_{4}=0.1$. Following the lowest line, we arrive at a critical chemical potential $\mu_{c}=0.02695$, around which there is a jump of the slop of the free energy as a function of $\mu$. That implies a first order flat space/boson star phase transition at $\mu_{c}$. We also study the behaviors of $\psi_{2}$ with $q=100, m^{2}=-2$ and $C_{4}=0.1$ in the right panel of figure 5. At the critical chemical potential $\mu_{c}=0.02695$, we have a jump of $\psi_{2}$. Compared with behaviors of the free energy, we again conclude that the jump of $\psi_{2}$ implies a first order phase transition. So we include that Stückelberg mechanism could trigger first order phase transitions in gravity systems in a box just as properties found in holographic AdS gravity systems. 

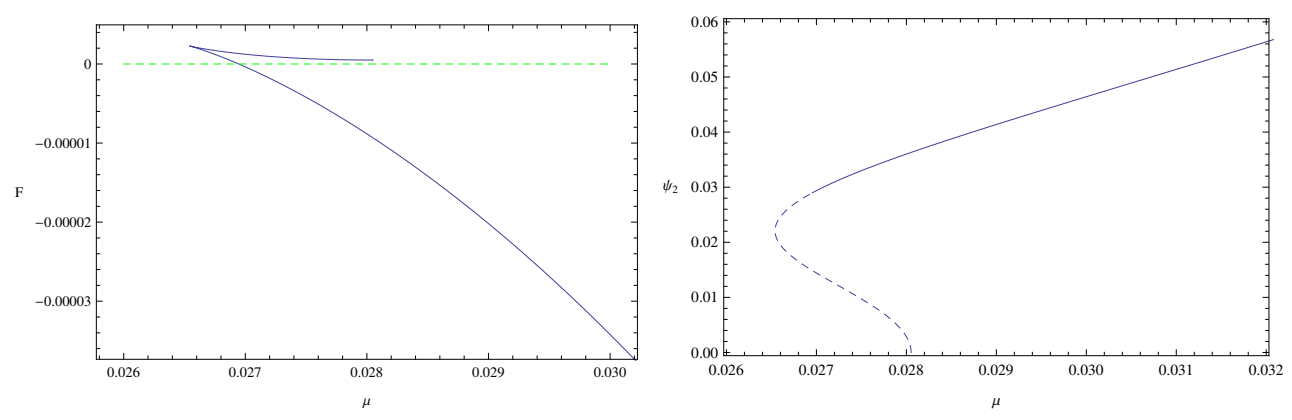

Figure 5. (Color online) The two panels are with $q=100, m^{2}=-2$ and $C_{4}=0.1$. We show behaviors of free energy of boson star phases with solid blue line in the left panel and the dashed green line of $F=0$ in the left panel is with the flat space. We also show behaviors of $\psi_{2}$ in the right panel. In addition, the solid blue curve in the right panel represents the stable phases and the dashed blue line in the right panel corresponds to unstable phases.
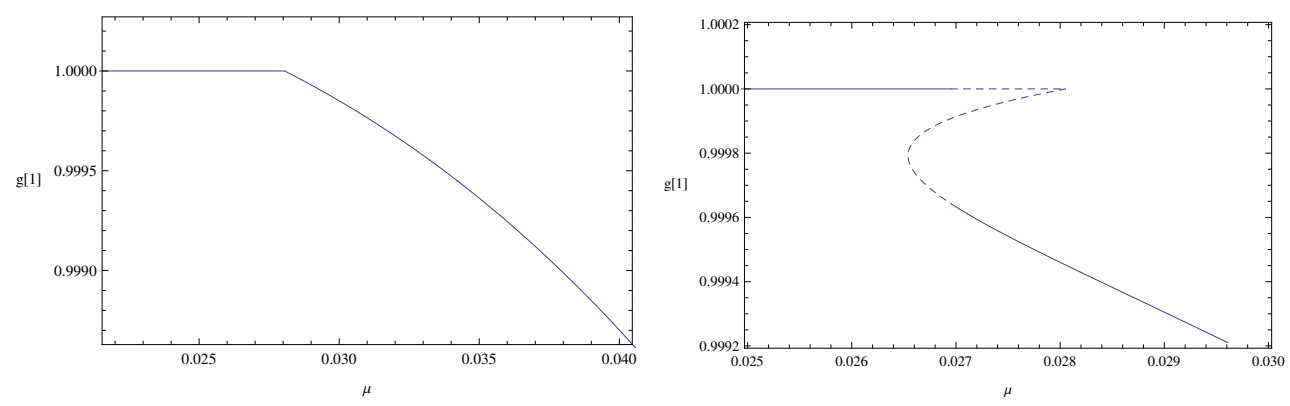

Figure 6. (Color online) We show $g(1)$ with respect to chemical potential $\mu$. In the left panel, the curve is with phases of $q=100, m^{2}=-2$ and $C_{4}=0$ and the right panel represents cases of $q=4$, $m^{2}=-2$ and $C_{4}=0.1$. We show stable phases with solid curves and unstable phases with dashed curves.

Besides the parameter $\psi_{2}$, we also find that the metric solutions can be used to disclose properties of phase transitions in our flat space/boson star transition model. In the left panel of figure 6 , when there are second order phase transitions with $q=100, m^{2}=-2$ and $C_{4}=0, g(1)$ has a jump of the slope with respect to chemical potential at the critical phase transition points $\mu_{c}=0.02806$. In contrast, for the case of first order phase transitions with $q=100, m^{2}=-2$ and $C_{4}=0.1$ in the right panel of figure $6, g(1)$ has a jump at the threshold value $\mu_{c}=0.02695$. Compared with results in figure 2 and figure 5 , we conclude that metric solutions can be used to determine the threshold phase transition points and the order of flat space/boson star phase transitions, where similar results have been found in holographic metal/superconductor models [47].

\section{Conclusions}

We studied a general four dimensional flat space/boson star transition model in a box beyond the probe limit. With numerical methods, we deduced the parameter $\psi_{2}$ from behaviors of the scalar field on the box boundary and found that $\psi_{2}$ can be used to detect 
the critical phase transition points and also the order of transitions. Similar to holographic insulator/superconductor transitions, we found that for large scalar charge $q>4.5$, there is only second order transitions and small charge $q<4.5$ could trigger first order phase transitions. For the second order transitions, we obtained an analytical relation $\psi_{2} \propto\left(\mu-\mu_{c}\right)^{1 / 2}$, which also holds in the holographic insulator/superconductor system in accordance with mean field theories. As a summary, we examined effects of the scalar mass on the second order critical chemical potential mainly from behaviors of $\psi_{2}$. We found the more negative scalar mass makes the second order phase transition more easier to happen qualitatively in accordance with cases in holographic insulator/superconductor models. For large scalar charge $q>4$.5, Stückelberg mechanism could make the transformation of the order of phase transitions from the second order into the first order and the metric solutions were also proved to be useful in detecting properties of transitions which are similar to cases in holographic theories. In summary, we obtained richer physics mainly through behaviors of $\psi_{2}$ in the generalized flat space/boson star transition model with box boundary conditions by including non-zero scalar mass, various scalar charge and Stückelberg mechanism. And we also pointed out that effects of scalar mass, scalar charge and Stückelberg mechanism on transitions in a box is qualitatively the same with those of holographic insulator/superconductor theories in AdS gravity and our results provided additional hints of the existence of similar holographic theories in gravity systems in a box.

\section{Acknowledgments}

We would like to thank the anonymous referee for the con-structive comments to improve the manuscript. This work was supported by the National Natural Science Foundation of China under Grant No. 11305097; the Shaanxi Province Science and Technology Department Foundation of China under Grant No. 2016JQ1039.

Open Access. This article is distributed under the terms of the Creative Commons Attribution License (CC-BY 4.0), which permits any use, distribution and reproduction in any medium, provided the original author(s) and source are credited.

\section{References}

[1] J.W. York, Black hole thermodynamics and the Euclidean Einstein action, Phys. Rev. D 33 (1986) 2092.

[2] H.W. Braden et al., Charged black hole in a grand canonical ensemble, Phys. Rev. D 42 (1990) 3376.

[3] S.W. Hawking and D.N. Page, Thermodynamics of Black Holes in anti-de Sitter Space, Commun. Math. Phys. 87 (1983) 577 [inSPIRE].

[4] J.M. Maldacena, The large- $N$ limit of superconformal field theories and supergravity, Int. J. Theor. Phys. 38 (1999) 1113 [hep-th/9711200] [INSPIRE].

[5] S.S. Gubser, I.R. Klebanov and A.M. Polyakov, Gauge theory correlators from noncritical string theory, Phys. Lett. B 428 (1998) 105 [hep-th/9802109] [INSPIRE]. 
[6] E. Witten, Anti-de Sitter space and holography, Adv. Theor. Math. Phys. 2 (1998) 253 [hep-th/9802150] [INSPIRE].

[7] S.A. Hartnoll, C.P. Herzog and G.T. Horowitz, Building a Holographic Superconductor, Phys. Rev. Lett. 101 (2008) 031601 [arXiv:0803.3295] [InSPIRE].

[8] S.A. Hartnoll, C.P. Herzog and G.T. Horowitz, Holographic Superconductors, JHEP 12 (2008) 015 [arXiv:0810.1563] [INSPIRE].

[9] G.T. Horowitz, Introduction to Holographic Superconductors, Lect. Notes Phys. 828 (2011) 313 [arXiv:1002.1722] [INSPIRE].

[10] R. Gregory, S. Kanno and J. Soda, Holographic Superconductors with Higher Curvature Corrections, JHEP 10 (2009) 010 [arXiv:0907.3203] [INSPIRE].

[11] L. Barclay, R. Gregory, S. Kanno and P. Sutcliffe, Gauss-Bonnet Holographic Superconductors, JHEP 12 (2010) 029 [arXiv: 1009.1991] [INSPIRE].

[12] T. Nishioka, S. Ryu and T. Takayanagi, Holographic Superconductor/Insulator Transition at Zero Temperature, JHEP 03 (2010) 131 [arXiv:0911.0962] [INSPIRE].

[13] R.-G. Cai, H.-F. Li and H.-Q. Zhang, Analytical Studies on Holographic Insulator/Superconductor Phase Transitions, Phys. Rev. D 83 (2011) 126007 [arXiv:1103.5568].

[14] H.B. Zeng, Y. Tian, Z.Y. Fan and C.-M. Chen, Nonlinear Transport in a Two Dimensional Holographic Superconductor, Phys. Rev. D 93 (2016) 121901 [arXiv:1604.08422].

[15] Y.-P. Hu, H.-F. Li, H.-B. Zeng and H.-Q. Zhang, Holographic Josephson Junction from Massive Gravity, Phys. Rev. D 93 (2016) 104009 [arXiv: 1512.07035].

[16] Y. Liu, Y. Gong and B. Wang, Non-equilibrium condensation process in holographic superconductor with nonlinear electrodynamics, JHEP 02 (2016) 116 [arXiv:1505.03603] [INSPIRE].

[17] X.-M. Kuang and E. Papantonopoulos, Building a Holographic Superconductor with a Scalar Field Coupled Kinematically to Einstein Tensor, JHEP 08 (2016) 161 [arXiv:1607.04928] [INSPIRE].

[18] F.Aprile and J.G. Russo, Models of holographic superconductivity, Phys. Rev. D 81 (2010) 026009 [arXiv:0912.0480].

[19] A. Salvio, Holographic Superfluids and Superconductors in Dilaton-Gravity, JHEP 09 (2012) 134 [arXiv: 1207.3800] [INSPIRE].

[20] J. Jing, Q. Pan and S. Chen, Holographic Superconductors with Power-Maxwell field, JHEP 11 (2011) 045 [arXiv: 1106.5181] [InSPIRE].

[21] J. Sonner, A Rotating Holographic Superconductor, Phys. Rev. D 80 (2009) 084031 [arXiv: 0903.0627].

[22] Y. Peng, Holographic entanglement entropy in superconductor phase transition with dark matter sector, Phys. Lett. B 750 (2015) 420 [arXiv:1507.07399].

[23] X.-H. Ge, B. Wang, S.-F. Wu and G.-H. Yang, Analytical study on holographic superconductors in external magnetic field, JHEP 08 (2010) 108 [arXiv:1002.4901] [INSPIRE].

[24] Y. Brihaye and B. Hartmann, Holographic superconductors in $3+1$ dimensions away from the probe limit, Phys. Rev. D 81 (2010) 126008. 
[25] P. Basu, J. He, A. Mukherjee, M. Rozali and H.H. Shieh, Competing Holographic Orders, JHEP 10 (2010) 092 [arXiv: 1007.3480] [INSPIRE].

[26] L.A. Pando Zayas and D. Reichmann, A Holographic Chiral $p_{x}+i p_{y}$ Superconductor, Phys. Rev. D 85 (2012) 106012 [arXiv:1108.4022].

[27] R.C. Myers and A. Singh, Comments on Holographic Entanglement Entropy and RG Flows, JHEP 04 (2012) 122 [arXiv:1202.2068] [INSPIRE].

[28] T. Albash and C.V. Johnson, Holographic Studies of Entanglement Entropy in Superconductors, JHEP 05 (2012) 079 [arXiv: 1202.2605] [INSPIRE].

[29] L. Yin, H.-c. Ren, T.K. Lee and D. Hou, Momentum Analyticity of Transverse Polarization Tensor in the Normal Phase of a Holographic Superconductor, JHEP 08 (2016) 116 [arXiv: 1605. 07547] [INSPIRE].

[30] D. Momeni, H. Gholizade, M. Raza and R. Myrzakulov, Holographic Entanglement Entropy in 2D Holographic Superconductor via $A d S_{3} / C F T_{2}$, Phys. Lett. B 747 (2015) 417 [arXiv: 1503.02896] [INSPIRE].

[31] Y. Ling, P. Liu and J.-P. Wu, Note on the butterfly effect in holographic superconductor models, Phys. Lett. B 768 (2017) 288 [arXiv: 1610.07146] [INSPIRE].

[32] R.M. Wald, The Thermodynamics of Black Holes, Living Rev. Rel. 4 (2001) 6 [gr-qc/9912119].

[33] P. Hut, Charged black holes and phase transitions, Mon. Not. Roy. Astron. Soc. 180 (1977) 379 [arXiv:0705.3269] [InSPIRE].

[34] G.W. Gibbons and M.J. Perry, Black Holes in Thermal Equilibrium, Phys. Rev. Lett. 36 (1976) 985.

[35] P. Basu, C. Krishnan and P.N.B. Subramanian, Hairy Black Holes in a Box, JHEP 11 (2016) 041 [arXiv: 1609.01208] [INSPIRE].

[36] P. Basu, C. Krishnan and P.N. Bala Subramanian, Phases of Global AdS Black Holes, JHEP 06 (2016) 139 [arXiv:1602.07211] [INSPIRE].

[37] G.T. Horowitz and B. Way, Complete Phase Diagrams for a Holographic Superconductor/Insulator System, JHEP 11 (2010) 011 [arXiv:1007.3714] [INSPIRE].

[38] G.T. Horowitz and M.M. Roberts, Holographic Superconductors with Various Condensates, Phys. Rev. D 81 (2008) 126008 [arXiv:0810.1077].

[39] Q. Pan, B. Wang, E. Papantonopoulos, J. Oliveira and A.B. Pavan, Holographic Superconductors with various condensates in Einstein-Gauss-Bonnet gravity, Phys. Rev. D 81 (2010) 106007 [arXiv:0912.2475].

[40] Y. Peng and Q. Pan, Holographic entanglement entropy in general holographic superconductor models, JHEP 06 (2014) 011 [arXiv: 1404.1659] [INSPIRE].

[41] S. Franco, A. Garcia-Garcia and D. Rodriguez-Gomez, A general class of holographic superconductors, JHEP 04 (2010) 092 [arXiv: 0906.1214] [INSPIRE].

[42] S. Franco, A.M. Garcia-Garcia and D. Rodriguez-Gomez, A holographic approach to phase transitions, Phys. Rev. D 81 (2010) 041901 [arXiv:0911.1354].

[43] Q. Pan and B. Wang, General holographic superconductor models with Gauss-Bonnet corrections, Phys. Lett. B 693 (2010) 159 [arXiv: 1005.4743] [INSPIRE]. 
[44] Y. Peng, Q. Pan and B. Wang, Various types of phase transitions in the AdS soliton background, Phys. Lett. B 699 (2011) 383 [arXiv:1104.2478] [INSPIRE].

[45] R.-G. Cai, S. He, L. Li and L.-F. Li, Entanglement Entropy and Wilson Loop in Stückelberg Holographic Insulator/Superconductor Model, JHEP 10 (2012) 107 [arXiv:1209.1019] [INSPIRE].

[46] Y. Peng and Q. Pan, Holographic entanglement entropy in general holographic superconductor models, JHEP 06 (2014) 011 [arXiv: 1404.1659] [INSPIRE].

[47] Y. Peng and Y. Liu, A general holographic metal/superconductor phase transition model, JHEP 02 (2015) 082 [arXiv: 1410.7234] [InSPIRE].

[48] S.R. Dolan, S. Ponglertsakul and E. Winstanley, Stability of black holes in Einstein-charged scalar field theory in a cavity, Phys. Rev. D 92 (2015) 124047 [arXiv:1507.02156].

[49] S. Ponglertsakul and E. Winstanley, Effect of scalar field mass on gravitating charged scalar solitons and black holes in a cavity, Phys. Lett. B 764 (2017) 87 [arXiv:1610.00135] [INSPIRE].

[50] N. Sanchis-Gual, J.C. Degollado, P.J. Montero, J.A. Font and C. Herdeiro, Explosion and Final State of an Unstable Reissner-Nordström Black Hole,

Phys. Rev. Lett. 116 (2016) 141101 [arXiv: 1512.05358] [INSPIRE]. 